\title{
Are COPD Prescription Patterns Aligned with Guidelines? Evidence from a Canadian Population-Based Study
}

This article was published in the following Dove Press journal: International Journal of Chronic Obstructive Pulmonary Disease

\author{
Taraneh Bahremand' \\ Mahyar Etminan ${ }^{2}$ \\ Nardin Roshan-Moniri ${ }^{1}$ \\ Mary A De Vera $\mathbb{D}^{\prime}$ \\ Hamid Tavakoli (iD) \\ Mohsen Sadatsafavi (iD) \\ 'Collaboration for Outcomes Research \\ and Evaluation, Faculty of Pharmaceutical \\ Sciences, The University of British \\ Columbia, Vancouver, BC, Canada; \\ ${ }^{2}$ Department of Ophthalmology, Faculty \\ of Medicine, The University of British \\ Columbia, Vancouver, BC, Canada
}

Background: In contemporary guidelines for the management of Chronic Obstructive Pulmonary Disease (COPD), the history of acute exacerbations plays an important role in the choice of long-term inhaled therapies. This study aimed at evaluating population-level trends of filled inhaled prescriptions over the time course of COPD and their relation to the history of exacerbations.

Methods: We used administrative health databases in British Columbia, Canada (1997-2015), to create a retrospective incident cohort of individuals with diagnosed COPD. We quantified long-acting inhaled medication prescriptions within each year of follow-up and documented their trend over the time course of COPD. Using generalized linear models, we investigated the association between the frequent exacerbator status ( $\geq 2$ moderate or $\geq 1$ severe exacerbation(s) in the previous 12 months) and filling a prescription after a physician visit.

Results: 132,004 COPD patients were included (mean age 68.6, 49.2\% female). The most common medication class during the first year of diagnosis was inhaled corticosteroids (ICS, used by $49.9 \%$ ), followed by long-acting beta-2 adrenoreceptor agonists (LABA, 31.8\%). Longacting muscarinic receptor antagonists (LAMA) were the least commonly prescribed (10.4\%). ICS remained the most common prescription throughout follow-up, being used by approximately $50 \%$ of patients during each year. $39.0 \%$ of patients received combination inhaled therapies in their first year of diagnosis, with ICS+LABA being the most common (30.7\%). The association with exacerbation history was the most pronounced for triple therapy with an odds ratio (OR) of 2.68 for general practitioners and 2.02 for specialists ( $<<0.001$ for both). Such associations were generally stronger among GPs compared with specialists, with the exception of monotherapy with LABA or ICS.

Conclusion: We documented low utilization of monotherapies (specifically LAMA) and high utilization of combination therapies (particularly ICS containing). Specialists were less likely to consider exacerbation history in the choice of inhaled therapies compared with GPs.

Keywords: COPD, prescription, medication, exacerbation, time trend
Correspondence: Mohsen Sadatsafavi Faculty of Pharmaceutical Sciences, University of British Columbia,

Vancouver, BC, Canada

Email msafavi@mail.ubc.ca

\section{Introduction}

Chronic obstructive pulmonary disease (COPD) is a common disease of the airways characterized by progressive airflow limitation and periods of intensified disease activity referred to as acute exacerbations (or lung attacks). ${ }^{1}$ COPD is among the leading causes of disease burden in terms of lost disability-adjusted life years (DALYs). ${ }^{2,3}$ In Canada, exacerbations of COPD are the leading cause of medical hospital admissions and a major cause of morbidity and mortality. ${ }^{4}$ 
In all stages of COPD, risk factor modification (eg, smoking cessation) has a high potential for modifying the course of the disease. However, for most patients, pharmacotherapy is an essential component of disease management. ${ }^{5}$ In contemporary COPD management guidelines, exacerbations are an important determinant of initiation and choice of drug therapy. For example, in the current Canadian Thoracic Society (CTS) guidelines, treatment choice is based on the dichotomization of patients into frequent or non-frequent exacerbators, ${ }^{6}$ with frequent exacerbator status defined based on 12-month history ( $\geq 2$ moderate or $\geq 1$ severe exacerbation(s)). In general, treatment is stepped up (intensified) if the patient becomes a frequent exacerbator, and can be stepped down if the patient is a non-frequent exacerbator. Other disease management strategies such as the influential Global Initiative for Chronic Obstructive Lung Disease $(G O L D)^{5}$ and the American Thoracic Society's guidelines ${ }^{7}$ adopt a similar approach.

Adherence to guideline recommendations on pharmacotherapies is generally low for chronic diseases. ${ }^{8}$ This might be due to the lack of awareness about guidelines by care providers, or low acceptance of, and adherence to, care providers' recommendations by patients. Evaluating the level of adherence to guidelines in "real world" settings enables the identification of preventable gaps in care. Population-based health databases that capture treatment patterns at the community level without the risk of Hawthorne effect (individuals modifying their behavior as a reaction to being observed) or recall bias are a unique resource for this purpose. The aims of the current study were to use such databases to describe the trend of filled prescriptions for inhaled medication throughout the time course of COPD (primary objective), and to evaluate the association between prescribed inhaled therapies with the frequent-exacerbator definition (secondary objective).

\section{Methods}

This study was approved by The University of British Columbia's human ethics board (\#H13-00684). All inferences, opinions, and conclusions drawn in this research are those of the authors and do not reflect the opinions or policies of the Data Steward(s). The health databases complied with the Freedom of Information and Protection of Privacy Act.

\section{Data Sources}

We used administrative health databases of British Columbia (a Canadian Province with a population of $5.07 \mathrm{M}$ as of
$2019^{9}$ ) from January 1997 to December 2015. The administrative needs of the Province's public healthcare system have resulted in the accumulation of healthcare encounter data for all legal residents of the Province. The data have high reliability with a very low rate of missing values. ${ }^{10} \mathrm{We}$ had access to demographics, ${ }^{11}$ hospitalization, ${ }^{12}$ outpatient services, ${ }^{13}$ and filled prescriptions ${ }^{14}$ records (including drug dose, quantity and day supply). Hospitalization and outpatient services records contain diagnostic International Classification of Diseases (ICD) codes. Each filled prescription record contains a unique identifier for the drug, as well as the dose and duration of supply. These data have frequently been used to quantity healthcare resource use patterns and their association with outcomes. ${ }^{15,16}$

\section{Study Population}

We created an incident cohort of individuals with diagnosed COPD using a previously validated case definition. ${ }^{17}$ In this definition, a patient is categorized as having COPD if during any 24-month rolling window, they had $\geq 1$ hospitalization or $\geq 2$ outpatient visits with COPD as the primary diagnosis (ICD-9: 491，492，493.2，496; ICD-10: J41-J44). In a previous review study, this definition had a sensitivity of $65.5 \%$ and a specificity of $91.5 \%$ (which is likely to be even higher in our sample given that individuals had to have COPD-related prescription records to contribute to the results). ${ }^{17}$ The lower bound for the age criteria was 35 , similar to previous studies ${ }^{18,19}$ to ensure that asthma patients (who might have similar health records as patients with COPD) were not over-represented. Patients entered the cohort on the date of their first COPD-related outpatient visit, which was considered the entry date, marking the beginning of follow-up. To ensure that an incident cohort of COPD patients is being selected, only those who were captured in the data for at least five years before their entry date were included. Patients were followed from their entry date to the date of their last resource use of any kind, death, end of registration in the database, or December 31st, 2015 (the administrative end of the study), whichever occurred first.

\section{Outcomes}

We evaluated two outcomes for the primary objective (illustrating medication trends over the time course of COPD). The first was filled prescriptions for long-acting inhaled medications during the time course of COPD. This was quantified in terms of the proportion of patients who filled prescriptions for a given medication class, as well as the average (per patient) dose-adjusted number of canisters 
dispensed. The following medication classes were evaluated: long-acting muscarinic receptor antagonists (LAMA), long-acting beta-2 adrenoreceptor agonists (LABA), and inhaled corticosteroids (ICS). We used a master list of COPD-related inhaled medications identified by their unique identifier linked with dose-equivalency tables (Supplementary Material - Section 1). The second outcome was filled prescriptions for combination therapies. The following combination therapies were considered: LAMA+LABA, LAMA $+\mathrm{ICS}, \mathrm{LABA}+\mathrm{ICS}$, and $\mathrm{LAMA}+\mathrm{LABA}+\mathrm{ICS}$. This outcome was quantified in terms of 1) the proportion of patients who, at least for part of the year, received inhaled therapies of different classes, and 2) medication possession ratio (MPR) for combination therapies. MPR was determined by counting the number of days within a follow-up year in which the patient was within the duration of supply of filled prescriptions for more than one class of medication (in a single inhaler or separate inhalers) and dividing it by the length of the follow-up period (365 days). If different classes of therapies had been dispensed for the patient in separate inhalers, they counted towards combination therapy if their duration of supply overlapped. For this (primary objective) we included all prescription records by general practitioners (GPs) and specialists, regardless of who prescribed them.

The outcome for the secondary objective (association between frequent-exacerbator status and inhaled medication prescription) was filled prescriptions within 14 days after a COPD-related outpatient visit to a physician. We limited the visits to GP, internal medicine specialist, and respirologist (the latter two are collectively referred to as specialists). The reason is that the clinical experts on our team believed that other specialties rarely initiate or change COPD-related medications and mostly refer the patient to their treating physician. If multiple visits occurred within 14 days of each other for the same patient, only the last visit was considered. At the time of a physician visit, a patient was classified as a frequent or non-frequent exacerbator based on their exacerbation history in the previous 12 months. We identified moderate and severe exacerbations using a previously published algorithm. ${ }^{20}$ A moderate exacerbation was defined based on filling prescriptions for oral corticosteroid or antibiotics within seven days after an outpatient visit with the main diagnostic (ICD) code of COPD (list of medications in Supplementary Material - Section 1). A severe exacerbation was defined as an instance of hospital admission with the main discharge diagnosis for COPD.

\section{Statistical Analysis}

For the primary objective, we divided the follow-up time of each patient into adjacent 12-month periods, starting from the entry date (if the last period was $<12$ months it was ignored). We plotted the proportion of COPD patients who received a given class of COPD medication as a function of years since COPD diagnosis. We also plotted the average number of dose-adjusted canisters per COPD patient during each follow-up period, separately for each inhaled medication class. In order to convert dispensed quantities to number of canisters, we used a specific formulation as the reference for each medication class, as follows: 1) salmeterol $50 \mathrm{mcg}$, 60 doses per canister for LABA, 2) beclomethasone 100 mcg, 200 doses per canister for ICS, and 3) tiotropium 18 mcg, 30 doses per canister for LAMA.

To test for time trends, we used a generalized linear model (with logit link function and binomial distribution) with generalized estimating equation to account for the clustering of observation units (patient-years) within patients. Years since the entry date was the independent variable, whose coefficient captured the time trend. We did not control for any other variable in this analysis given the primary interest was examining temporal trends.

For the secondary objective, we used a generalized linear model (with the same link function and distribution as above) to associate filled prescriptions after physician visits with exacerbation history. This model was fitted separately for each medication class, and for GPs versus specialists. Again, we used a generalized estimating equation to account for the clustered nature of the data (multiple physician visits for each patient). We controlled for the following variables: sex, age on the date of visit, and socio-economic status on the date of visit (estimated based on neighbourhood income quantiles). SAS Enterprise Guide (version 7.1, Cary, NC, USA) was used for all analyses. Two-tailed p-values were considered significant at 0.05 level.

We performed a sensitivity analysis that explored whether our findings are affected by the inadvertent inclusion of some patients with asthma but without fixed airway obstruction. For this analysis, we excluded all individuals who met a previously validated case definition of asthma ${ }^{21}$ any time during the study period (before or after COPD diagnosis). We reported the proportion of each medication 
classes that were prescribed after a physician visit, separately by the frequent exacerbator status.

\section{Results}

In our study, 132,004 individuals satisfied the case definition of COPD. Of these, 64,942 patients (49.2\%) were female and the mean age at entry was $68.6(\mathrm{SD}=12.5)$ years. These patients contributed 707,575 years of follow-up (mean follow-up 5.4 years, $\mathrm{SD}=3.5$ ). Table 1 pro-

Table I Characteristics of Patients and the Visits in the Cohort

\begin{tabular}{|c|c|}
\hline $\begin{array}{l}\text { Patient Characteristics at Index } \\
\text { Date }\end{array}$ & $\begin{array}{l}\text { Study Cohort } \\
(n=132,004)\end{array}$ \\
\hline Female sex (\%) ${ }^{a}$ & $64,942(49.2)$ \\
\hline Age (SD) & $68.6(12.5)$ \\
\hline Years from COPD diagnosis & $5.4(3.5)$ \\
\hline Socioeconomic status (\%) & \\
\hline Quantile I & $34,699(26.3 \%)$ \\
\hline Quantile 2 & $28,116(21.3 \%)$ \\
\hline Quantile 3 & $24,630(18.7 \%)$ \\
\hline Quantile 4 & $22,500(17.1 \%)$ \\
\hline Quantile 5 & 19,545 (14.8\%) \\
\hline Unknown & $2,514(1.9 \%)$ \\
\hline $\begin{array}{l}\text { Visit Characteristics for the Sub- } \\
\text { Cohort of COPD Patients Alive } \\
\text { in } 2010\end{array}$ & $\begin{array}{l}\text { Visits Followed by } \\
\text { Prescription } \\
(n=260,164)\end{array}$ \\
\hline $\begin{array}{l}\text { Patient was frequent exacerbator in } \\
\text { the previous } 12 \text { months }\end{array}$ & $79,820(30.7 \%)$ \\
\hline History of PFT before visit & $228,464(87.8 \%)$ \\
\hline $\begin{array}{l}\text { History of asthma diagnosis before } \\
\text { visits }\end{array}$ & I 42,457 (54.8\%) \\
\hline $\begin{array}{l}\text { COPD-related medications in the } \\
\text { three months before the visit }\end{array}$ & \\
\hline ICS only & 28,624 (II.0\%) \\
\hline LABA only & $2,039(0.78 \%)$ \\
\hline LAMA only & $|8,4| 4(7.08 \%)$ \\
\hline LAMA+LABA & $2,086(0.8 \%)$ \\
\hline ICS+LABA & $80,205(30.83 \%)$ \\
\hline ICS+LAMA & 4,861 (1.87\%) \\
\hline ICS+LAMA+LABA & $71,152(27.35 \%)$ \\
\hline
\end{tabular}

Note: ${ }^{\mathrm{a}}$ Number of patients (and percent of cohort) are reported unless otherwise indicated.

Abbreviations: COPD, chronic obstructive pulmonary disease; PFT, pulmonary function test; ICS, inhaled corticosteroids; LABA, long-acting beta-2 adrenoceptor agonists; LAMA, long-acting muscarinic receptor antagonists. vides basic sociodemographic characteristics of these patients.

\section{Trends of Filled Prescriptions for Inhaled Medications Over the Time Course of COPD}

The trends are depicted in Figure 1. ICS was the most common medication class prescribed to patients in their first year of diagnosis $(49.9 \%$ - panel A). It was followed by LABA $(31.8 \%)$ and LAMA (10.4\%). The percentage of patients who filled ICS-containing prescriptions per year remained the same $(\sim 50 \%)$ throughout the time course of COPD, whereas this percentage increased modestly for LABA and LAMA (by $7 \%$ and $9 \%$, respectively, over 15 years). In terms of the quantity of dispensed medications, again ICS was the most prescribed ingredient (average of 2.9 canisters per patient per year over the 15-year period - panel B). The average number of canisters (per year) for LAMA, LABA, and ICS increased by $119 \%, 56 \%$, and $40 \%$, respectively, over 15 years.

The trends for combination therapies are illustrated in Figure 2. In the first year of diagnosis, $30.7 \%$ of patients received ICS+LABA (panel A), and on average $11.0 \%$ of a COPD patient-times was covered by these combination therapies (panel B). The second most common combination therapy was triple therapy (LAMA+LABA+ICS), taken by $6.1 \%$ of patients in their first year of diagnosis, covering $2.0 \%$ of their time in the first follow-up year. Other patterns of combination therapies were not common. Among patients with 12-15 years of COPD history, on average $58.5 \%$ received combination therapies, with MPR of $33.0 \%$. Both the proportion of patients receiving combination therapies and the average MPR increased for ICS + LABA and triple therapy over the time course of COPD.

\section{Association Between Filled Prescriptions After an Outpatient Visit and Exacerbation History}

During follow-up, 260,164 eligible outpatient visits were recorded that were followed with a filled prescription for an inhaled medication. $85 \%$ of the outpatient visits were made to GPs. In 79,820 (30.7\%) of visits, the patient was classified as frequent-exacerbator given their 12-month exacerbation history (Table 1). 

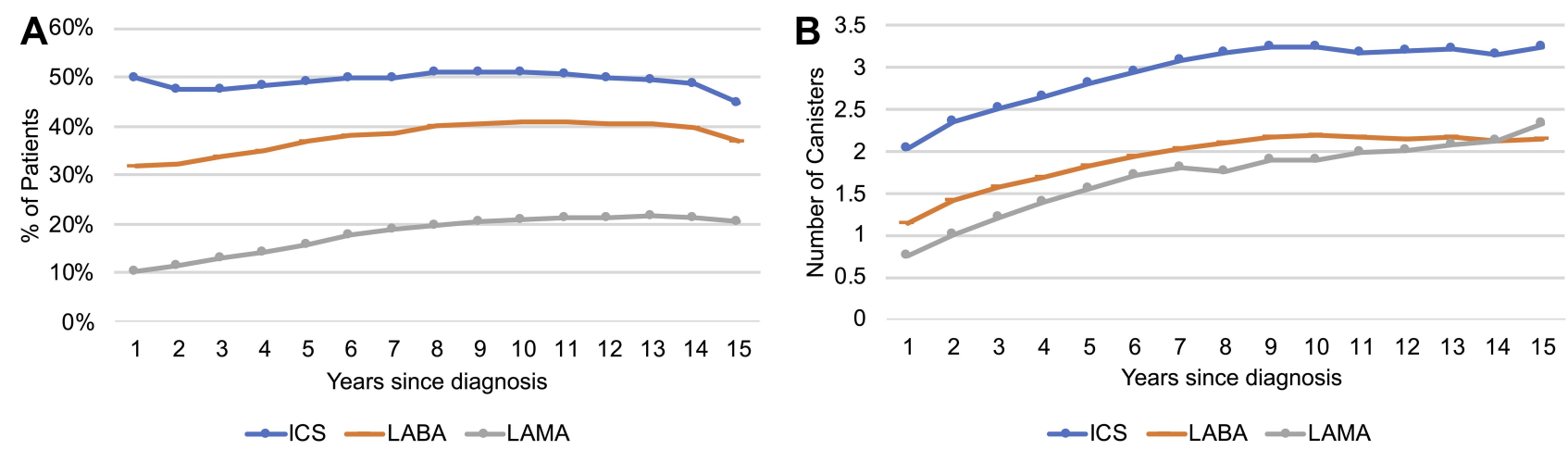

Figure I Trends in the proportion of patients filling at least one prescription (A) and average dose-adjusted number of canisters (B) for major COPD inhaled therapies, during disease course.

Abbreviations: ICS, inhaled corticosteroids; LABA, long-acting beta-2 adrenoceptor agonists; LAMA, long-acting muscarinic receptor antagonists.
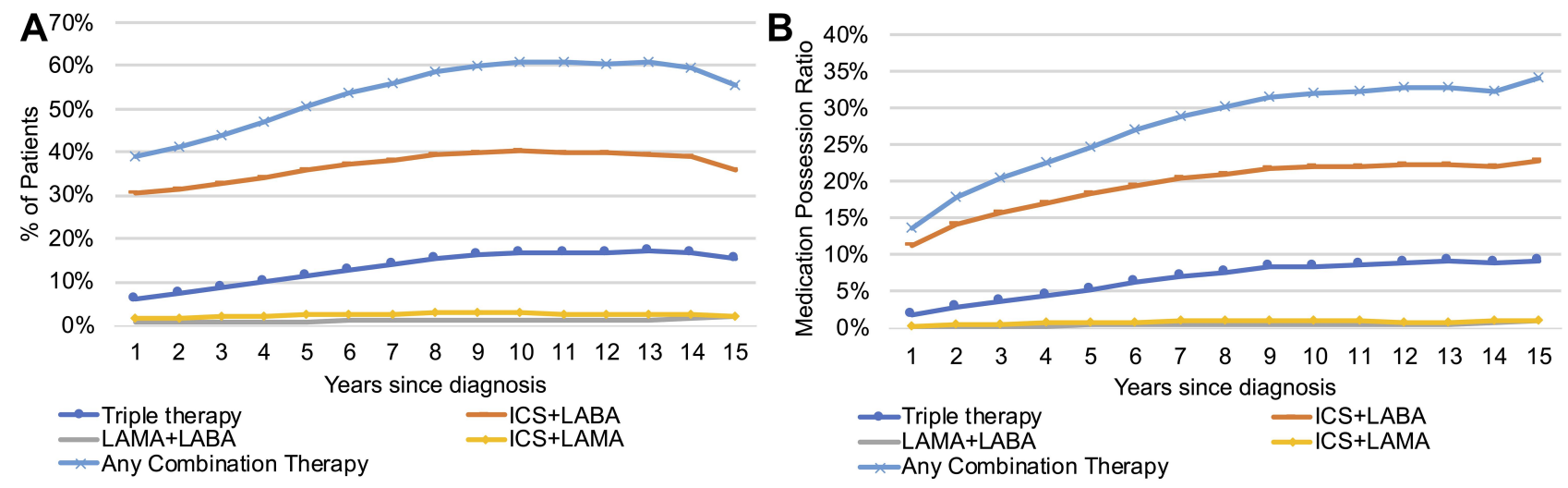

Figure 2 Trends in the proportion of patients on combination therapies (A) and average medication possession ratio (B) over the time course of COPD. Abbreviations: ICS, inhaled corticosteroids; LABA, long-acting beta-2 adrenoceptor agonists; LAMA, long-acting muscarinic agents; ICS/LABA/LAMA, triple therapy.

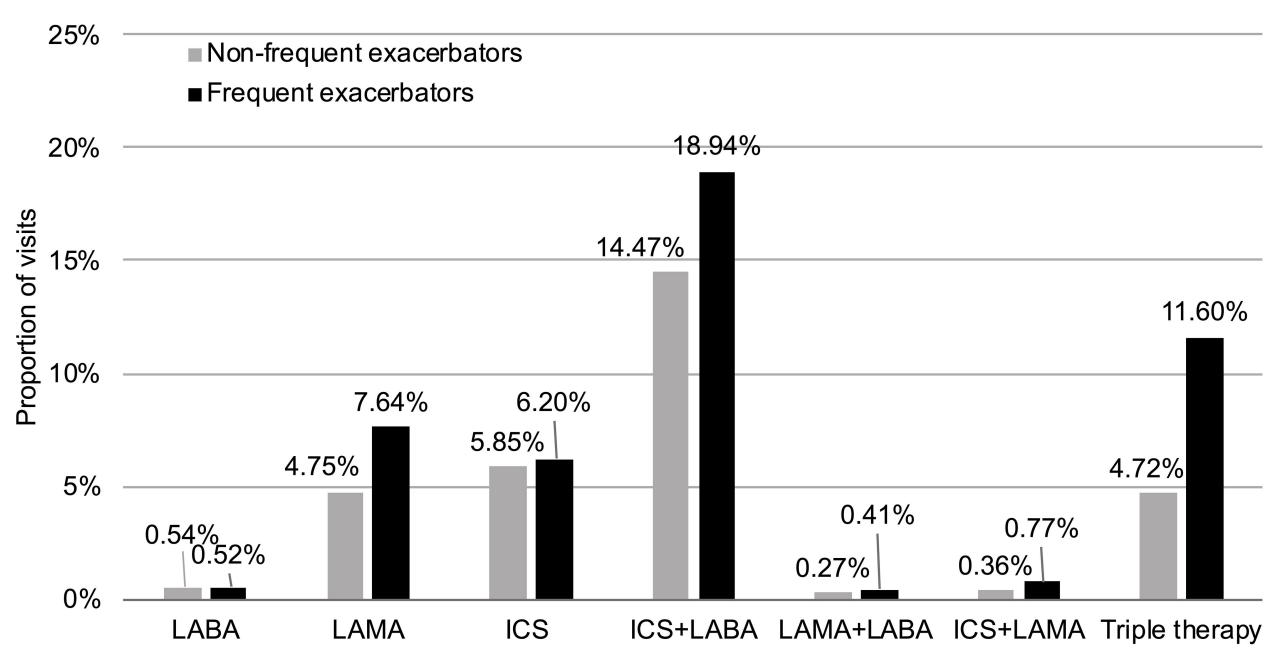

Figure 3 Frequency of long-acting inhaled medication prescription in frequent (dark bars) and non-frequent (light bars) exacerbators.

Abbreviations: ICS, inhaled corticosteroids; LABA, long-acting beta-2 adrenoceptor agonists; LAMA, long-acting muscarinic receptor antagonists. 
The frequencies (by percentage) of different inhaled therapies prescribed for COPD patients, stratified by the frequent exacerbator status, are provided in Figure 3. For non-frequent exacerbators, the most common treatment was ICS+LABA (14.5\%), followed by ICS alone (5.8\%). Outpatient visits among frequent exacerbators were most commonly followed by filled prescriptions for ICS+LABA (18.9\%) and triple therapy (11.6\%).

Figure 4 provides the adjusted odds ratios (ORs) and 95\% confidence intervals (CI) associating filled prescriptions with the frequent exacerbator status (compared to non-frequent exacerbators), separately for GPs and specialists. All associations were significant, except for two: LABA+LAMA therapy for specialists, and monotherapy with LABA for GPs. For both groups, triple therapy showed the strongest association with a positive exacerbation history (OR of 2.68 for GPs and 2.02 for specialists; $\mathrm{p}<0.0001$ for both). The associations were generally stronger (further away than 1.00) for GPs, with the exception of monotherapies with ICS or LABA.
The result of the sensitivity analysis is provided in Supplementary Material - Section 2, Supplementary Figure 1. The trend of medication prescription did not differ substantially from the main analysis, nor did the association between frequent exacerbator definition and prescription patterns. Again, ICS+LABA was the most common combination therapy after a physician visit, regardless of exacerbation history. Similar to the main analysis, the second most common therapy was monotherapy with ICS among non-frequent exacerbators and triple therapy among frequent exacerbators.

\section{Discussion}

Using 15 years of population-based data, we quantified the pattern of long-acting inhaled therapies in an incident cohort of patients with diagnosed COPD. We demonstrated that ICS was the most commonly prescribed medication class throughout the time course of the disease, with about $50 \%$ of patients having filled prescriptions for ICS during their first year of diagnosis. While this

\section{Odds Ratio for Medication Prescription in Frequent Exacerbators Compared to Non-frequent Exacerbators}

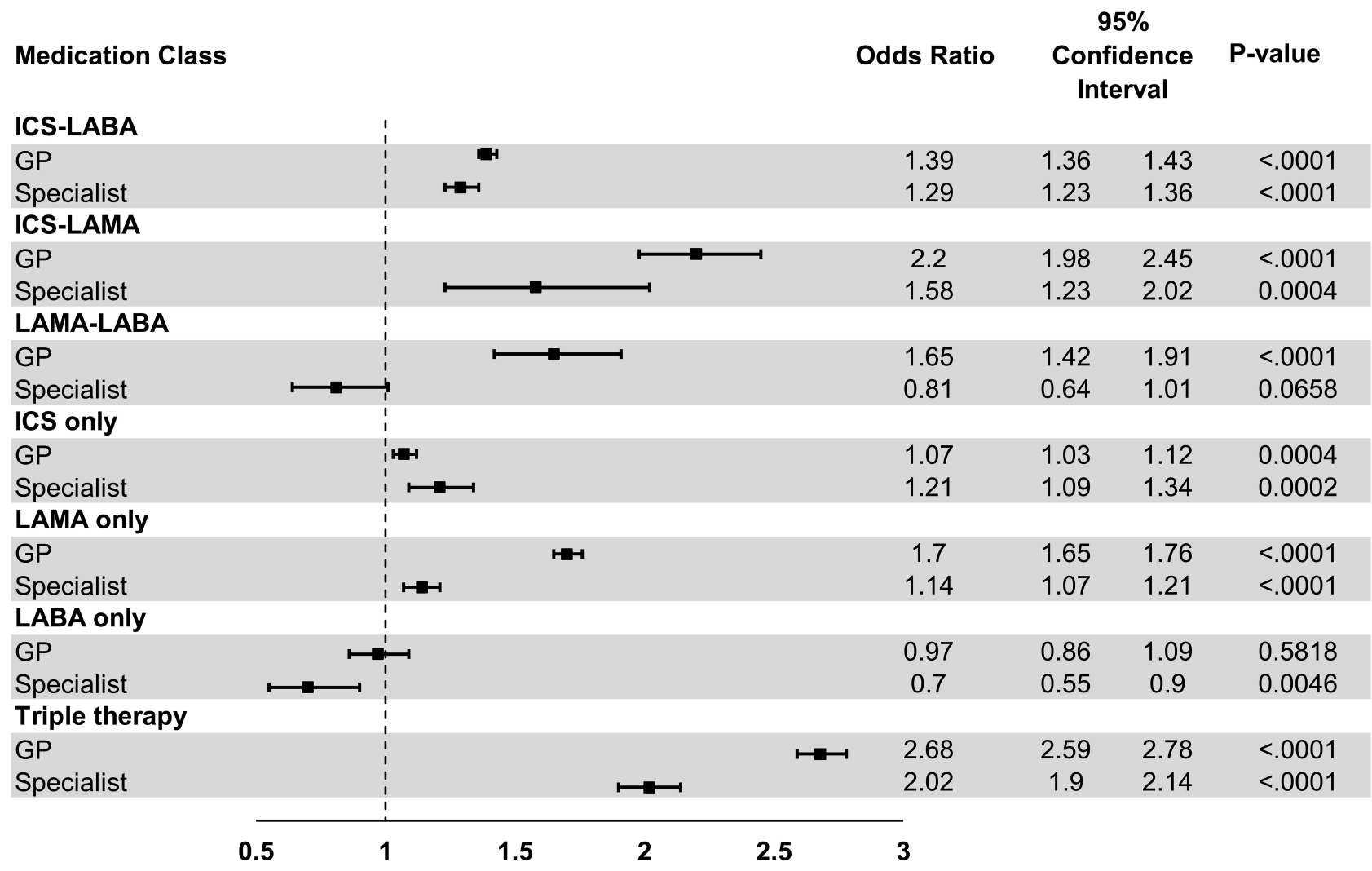

Figure 4 Forest plot of odds ratio (OR) and $95 \%$ confidence interval between frequent-exacerbator status and filled prescriptions for each medication type, separately for $\mathrm{GP}$ and specialist compared to non-frequent exacerbators.

Abbreviations: ICS, inhaled corticosteroids; LABA, long-acting beta-2 adrenoceptor agonists; LAMA, long-acting muscarinic receptor antagonists; GP, general practitioner. 
proportion remained roughly the same over the subsequent follow-up years, the average number of canisters of ICS per patient increased, indicating that the dosage of ICS increased over time among ICS users. The second most commonly used ingredient was LABA, received by a third of the cohort upon diagnosis. Further, we documented that $39.0 \%$ of patients received combination therapies in their first year of diagnosis, with ICS+LABA being the most common (30.7\%), followed by triple therapy. In the Canadian guidelines, the recommended first-line therapy is monotherapy with LAMA or LABA, and ICS should only be used among individuals who do not respond to dual LABA+LAMA therapy. ICS can be started earlier in the course of the disease in patients with a history of asthma. However, as our sensitivity analysis indicates, the use of ICS was also common in COPD patients without any history of asthma. As such, these results demonstrate potentially significant departures from guideline recommendations, in particular the overuse of ICS and combination therapies.

In addition, our results revealed a difference between GPs and specialists in terms of considering exacerbation history in prescribing inhalers. The Canadian (and GOLD) guidelines generally recommend intensifying medications (eg, adding a second or third therapy) among patients who experience exacerbations in the previous 12 months. Following this recommendation should make intensive treatments (dual therapies and triple therapy) positively associated with exacerbation history. Our results suggest that GPs are more responsive in their treatment choices to the positive exacerbation history. On the other hand, following guidelines should cause monotherapies to have a negative association with the frequent exacerbator status, as no patient with frequent exacerbations in the previous year should be on monotherapy. However, there was only one association for monotherapies that was negative (LABA monotherapy among specialists). Overall, this might suggest that treatment step-downs are not generally adhered to among GPs and specialists alike.

Our findings are similar to those of the previous studies which have shown that many prescriptions of ICS do not have clear indications. ${ }^{16,23}$ A study from the Province of Manitoba, Canada (for the 1997-2012 period), reported that ICS was by far the most commonly prescribed firstline therapy in patients with COPD $(28.2 \%)$, while LAMA $(2.1 \%)$ and LABA $(2.3 \%)$ were prescribed much less frequently. The proportion of patients who received ICS, which was often in combination with LABA, increased from $23.5 \%$ to $34.4 \%$ during the study period. ${ }^{18}$ Similar results have also been documented in two other Canadian provinces. ${ }^{24}$ Similar to our results, a UK-based study also reported overuse of ICS and undertreatment with LAMA + LABA. ${ }^{25}$

We are not aware of any previous study that correlates the choice of medication with the frequent exacerbator status and specialty of care. Previous studies have compared the differences in GP and respiratory specialist in terms of post-exacerbation management and outcomes, with one showing that although there were no differences in the 12-month re-admission and mortality rates between patients, there was significant difference in the management of acute exacerbations between the two groups. ${ }^{22}$ One potential mechanism explaining the seemingly higher adherence to guidelines among GPs in our study is that generally more complicated patients, such as those with more intensive symptoms, unsatisfactory response to previous therapies, and higher burden of comorbidity, are referred to specialists. This might result in specialists having to consider several different factors in their choice of treatment, not just what guidelines recommend. Another possible factor is the local healthcare policies. In British Columbia, both LAMA and ICS+LABA are "special authority" medications for GPs, requiring GPs to objectively document fixed airflow obstruction before being able to prescribe such medications. Obtaining a special authority for such prescriptions after an exacerbation might have been easier for GPs (eg, if the patient already received spirometry during their admission or visit to Emergency Room), making the prescriptions to be more strongly correlated with exacerbation history. Overall, the purpose of this study was not to explore the etiology of non-adherence to treatment recommendations. A study that addresses the reasons for the difference in treatment decisions between GPs and specialists is an important one that requires a dedicated design and analysis.

This study has multiple strengths. The use of data from the entire population of a well-defined geographic region reduces the risk of bias due to self-selection into the cohort. Retrieval of filled prescription records eliminates the risk of information or recall bias or Hawthorne effect. Avoiding recall bias is particularly important for the analysis of the associations between medication use and exacerbation history, as a history of exacerbation might affect patient recall. The large size of the data and several years of follow-up enabled us to have high statistical power to evaluate long-term trends. On the other hand, 
the limitations of this study should also be acknowledged. Filled prescription records do not necessarily equate to the actual medication that was recommended to the patient, as physicians might advise their patients to continue using previously stocked medications. Some physicians might also provide medication samples to patients that are not recorded in the data. One possible bias in this study is the under-reporting of exacerbations. Our algorithm for eliciting exacerbation history might not be completely aligned with physician assessment of exacerbation history. ${ }^{20}$

\section{Conclusion}

Taken together, these results suggest that the utilization of inhaled therapies for COPD is not aligned with current Canadian or international guidelines. This is most clearly demonstrated in the low utilization of LAMA and high utilization of ICS in newly diagnosed patients, as well as high utilization of combination therapies as first-line. An interesting finding of our study was that, compared to treatment recommendations by specialists, treatment recommendations made by GPs were potentially more reflective of the exacerbation history, but this might well reflect the more complicated clinical scenarios that specialists manage. Overall, improving adherence to guideline recommendations should be promoted across all specialty groups. Our results also suggest that treatments are not stepped down as recommended by guidelines, potentially resulting in significant levels of overtreatment.

\section{Author Contributions}

All authors made a significant contribution to the work reported, whether that is in the conception, study design, execution, acquisition of data, analysis and interpretation, or in all these areas; took part in drafting, revising or critically reviewing the article; gave final approval of the version to be published; have agreed on the journal to which the article has been submitted; and agree to be accountable for all aspects of the work.

\section{Funding}

This study was funded by the Canadian Respiratory Research Network.

\section{Disclosure}

MS has received speaker fees and honoraria from Boehringer Ingelheim, GlaxoSmithKline, and AstraZeneca. He has received research funds directly into his research accounts within The University of British
Columbia from Boehringer Ingelheim and AstraZeneca. The authors report no other conflicts of interest in this work.

\section{References}

1. Qureshi H, Sharafkhaneh A, Hanania NA. Chronic obstructive pulmonary disease exacerbations: latest evidence and clinical implications. Ther Advan Chronic Dis. 2014;5(5):212-227. doi: $10.1177 / 2040622314532862$

2. Collaborators CRD. GBD 2015 Chronic Respiratory Disease Collaborators Global, regional, and national deaths, prevalence, disability-adjusted life years, and years lived with disability for chronic obstructive pulmonary disease and asthma, 1990-2015: a systematic analysis for the Global Burden of Disease Study 2015. Lancet Respir Med. 2017;5:691-706.

3. WHO. Burden of COPD. Avialable from: https://www.who.int/ respiratory/copd/burden/en/. Accessed May 14, 2020.

4. Gershon AS, Guan J, Victor JC, Goldstein R, To T. Quantifying health services use for chronic obstructive pulmonary disease. Am J Respir Crit Care Med. 2013;187(6):596-601. doi:10.1164/rccm.201211-2044OC

5. Singh D, Agusti A, Anzueto A, et al. Global strategy for the diagnosis, management, and prevention of Chronic Obstructive Lung Disease: the GOLD science committee report 2019. Eur Respir J. 2019;53(5):1900164. doi:10.1183/13993003.00164-2019

6. Bourbeau J, Bhutani M, Hernandez P, et al. Canadian Thoracic Society Clinical Practice Guideline on pharmacotherapy in patients with COPD - 2019 update of evidence. Can J Respir Crit Care Sleep Med. 2019;3(4):210-232. doi:10.1080/24745332.2019.1668652

7. Pharmacologic Management of Chronic Obstructive Pulmonary DiseaseAugust. An official American Thoracic Society clinical practice guideline. Am J Respir Crit Care Med. 2020. https://www.atsjour nals.org/doi/10.1164/rccm.202003-0625ST.

8. Cantrell CR, Priest JL, Cook CL, Fincham J, Burch SP. Adherence to treatment guidelines and therapeutic regimens: a US claims-based benchmark of a commercial population. Popul Health Manage. 2011;14(1):33-41. doi:10.1089/pop.2010.0018

9. Services M of C. Population Estimates - Province of British Columbia. Available from: https://www2.gov.bc.ca/gov/content/data/ statistics/people-population-community/population/population-esti mates. Accessed July 22, 2020.

10. Williams JI, Young W. Inventory of Studies on the Accuracy of Canadian Health Administrative Databases. ICES; 1996.

11. British Columbia Ministry of Health [creator] (2013): Consolidation File (MSP Registration \& Premium Billing). Population Data BC [publisher]. Data Extract. MOH. Available from: http://www.pop data.bc.ca/data. Accessed March 15, 2021.

12. Canadian Institute for Health Information [creator].Discharge Abstract Database (Hospital Separations). Population Data BC [Publisher]. Data Extract. MOH; 2014. Available from: http:// www.popdata.bc.ca/data.

13. British Columbia. Ministry of Health Services MSP Fee-For-Service Payment Analysis | BC Government Publication | Legislative Library | Legislative Assembly of British Columbia. Available from: http:// www.llbc.leg.bc.ca/public/pubdocs/bcdocs/350351/index.htm>. Accessed March 15, 2021.

14. BC Ministry of Health [creator] (2014): PharmaNet. BC Ministry of Health [publisher]. Data Extract. Data Stewardship Committee (2013). Available from: http://www.popdata.bc.ca/data. Accessed March 15, 2021.

15. Kendzerska T, Leung R, Sandoz J, Gershon AS. C110 OSA OVERLAPS: ASTHMA, COPD, OBESITY HYPOVENTILATION SYNDROME, AND LUNG CANCER: modifiable effect of co-existence of chronic obstructive pulmonary disease and obstructive sleep apnea: evidence from clinical and health administrative data. Am J Respir Crit Care Med. 2017;195. 
16. Tavakoli H, Johnson KM, FitzGerald JM, et al. Trends in prescriptions and costs of inhaled medications in chronic obstructive pulmonary disease: a 19-year population-based study from Canada. Int J Chronic Obstruct Pulmonary Dis. 2019;14:2003. doi:10.2147/ COPD.S210897

17. Gershon AS, Wang C, Guan J, Vasilevska-Ristovska J, Cicutto L, To T. Identifying individuals with physician diagnosed COPD in health administrative databases. COPD. 2009;6(5):388-394. doi:10.1080/15412550903140865

18. Falk J, Dik N, Bugden S. An evaluation of early medication use for COPD: a population-based cohort study. Int J Chron Obstruct Pulmon Dis. 2016;11:3101-3108. doi:10.2147/COPD.S123643

19. Fabricius P, Løkke A, Marott JL, Vestbo J, Lange P. Prevalence of COPD in Copenhagen. Respiratory Medicine. 2011;105(3):410-417. doi:10.1016/j.rmed.2010.09.019

20. Tavakoli H, Chen W, Sin DD, FitzGerald JM, Sadatsafavi M. Predicting Severe COPD Exacerbations: developing a Population Surveillance Approach with Administrative Data. Annals ATS. 2020;17(9):1069-1076. doi:10.1513/AnnalsATS.202001-070OC
21. Gershon AS, Wang C, Guan J, Vasilevska-Ristovska J, Cicutto L, To T. Identifying patients with physician-diagnosed asthma in health administrative databases. Can Respir J. doi:10.1155/2009/963098

22. Wijayaratne K, Wilson J, Sivakumaran P, Sriram KB. Differences in care between general medicine and respiratory specialists in the management of patients hospitalized for acute exacerbations of chronic obstructive pulmonary disease. Ann Thorac Med. 2013;8 (4):197-203. doi:10.4103/1817-1737.118499

23. White P, Thornton H, Pinnock H, Georgopoulou S, Booth HP. Overtreatment of COPD with Inhaled Corticosteroids - Implications for Safety and Costs: cross-Sectional Observational Study. PLoS One. 2013;8(10):e75221. doi:10.1371/journal.pone.0075221

24. Bourbeau J, Sebaldt RJ, Day A, et al. Practice patterns in the management of chronic obstructive pulmonary disease in primary practice: the case study. Can Respir J. doi:10.1155/2008/173904

25. Raluy-Callado M, Lambrelli D, MacLachlan S, Khalid JM. Epidemiology, severity, and treatment of chronic obstructive pulmonary disease in the United Kingdom by GOLD 2013. Int $J$ Chron Obstruct Pulmon Dis. 2015;10:925-937. doi:10.2147/COPD.S82064

\section{Publish your work in this journal}

The International Journal of COPD is an international, peer-reviewed journal of therapeutics and pharmacology focusing on concise rapid reporting of clinical studies and reviews in COPD. Special focus is given to the pathophysiological processes underlying the disease, intervention programs, patient focused education, and self management protocols. This journal is indexed on PubMed Central, MedLine and CAS. The manuscript management system is completely online and includes a very quick and fair peer-review system, which is all easy to use. Visit http://www.dovepress.com/testimonials.php to read real quotes from published authors. 